\title{
Evaluation of an overnight non-culture test for detection of viable Gram-negative bacteria in endoscope channels
}

\section{다 (1) $\odot$}

Authors

Harminder Singh ${ }^{1}$, Donald R. Duerksen ${ }^{1}$, Gale Schultz ${ }^{2}$, Carol Reidy ${ }^{3}$, Pat DeGagne ${ }^{4}$, Nancy Olson ${ }^{4}$, Zoann Nugent ${ }^{1}$, Michelle J. Alfa ${ }^{4,5}$

Institutions

1 Dept of Internal Medicine, University of Manitoba, Winnipeg MB, Canada

2 Winnipeg Regional Health Authority, Winnipeg MB, Canada

3 St. Boniface Hospital, Winnipeg MB, Canada

4 St. Boniface Research Centre, Winnipeg, MB, Canada

5 Dept of Medical Microbiology, University of Manitoba, Winnipeg, MB, Canada

submitted 24.6.2018

accepted after revision 8.10 .2018

Bibliography

DOI https://doi.org/10.1055/a-0808-4342 |

Endoscopy International Open 2019; 07: E268-E273

(c) Georg Thieme Verlag KG Stuttgart · New York

ISSN 2364-3722

Corresponding author

Dr. Michelle Alfa, Ph.D., FCCM, Professor, Dept of Medical

Microbiology, University of Manitoba, Winnipeg, MB,

CANADA R3E 0J9

Fax: +1-204-789-3926

michellealfa001@gmail.com

\section{ABSTRACT}

Background and study aims Prevention of infection transmission from contaminated endoscopes would benefit from a rapid test that could detect low levels of viable bac- teria after high level disinfection. The aim of this study was to evaluate the rapid NOW! (RN) test's ability to detect endoscope contamination.

Materials and methods The RN test kit and the accompanying fluorometer were evaluated. The manufacturer states that a fluorometer signal $>300$ units is indicative of viable Gram-negative bacteria. Suspension testing of varying concentrations of Escherichia coli, Pseudomonas aeruginosa and Enterococcus faecalis were used to determine the RN test limit of detection. Simulated-use testing was done using a duodenoscope inoculated with $10 \%$ blood containing approximately 35 CFU E. coli per channel. Samples were extracted from the duodenoscope instrument channel and tested using the manufacturer's instructions.

Results The RN test could consistently detect 10 CFU of $E$. coli and P. aeruginosa (fluorescent signal of 9,000 to 11,000 units) but not $E$. faecalis. Sensitivity and specificity for Gram-negative bacteria were $93 \%$ and $90 \%$, respectively, using all of the suspensions in the study. Extraction of E. coli from an inoculated duodenoscope instrument channel repeatedly provided a positive signal (i.e. > 2,000 units). Conclusions The RN test can reliably detect low levels of Gram-negative bacteria in suspension as well as from samples extracted from endoscope channels. These preliminary findings are encouraging but further assessment of extraction efficacy, impact of organic residuals and clinical workflow are still needed.

\section{Introduction}

Transmission of multi-antibiotic-resistant bacteria from contaminated endoscopes has prompted healthcare facilities to culture their endoscopes in an attempt to detect contamination of organisms of high concern so that these endoscopes can be removed from use and reprocessed to eliminate infection transmission [1 - 7]. The newly released US Food and Drug
Administration/Centers for Disease Control and Prevention/ American Society for Microbiology (FDA/CDC/ASM) duodenoscope sample collection and culture protocol has been validated by the endoscope manufacturers and provides a standardized approach to culture duodenoscopes [8].

Unlike endoscope culture protocols from other countries, the FDA/CDC/ASM culture protocol is similar to that of Ross et al. (2015) [3] and indicates that ideally once a culture sample has been taken, the endoscope should be quarantined until 
the results of the culture are known, so that if the endoscope was contaminated, patient exposure during subsequent procedures could be avoided [8]. However, this culture and quarantine approach severely limits the number of clinical procedures that can be performed. Instead of being able to use an endoscope multiple times each day, with the quarantine process, it can only be used for one procedure every 2 to 3 days (depending on how long the culture results take). Culture of endoscopes is also fraught with additional standardization issues such as the sample collection method used (i.e. type of friction, type of extraction fluid, use of neutralizer) [9-13] and the culture protocol used (i. e. concentration of sample, culture media and duration of incubation) affect the sensitivity of cultures [9-17]. Although culture is recommended in many countries as a monitoring tool for endoscope reprocessing, [11, 18-19] it is not currently recommended in United States guidelines [20-22] and there is evidence that there can be false-negative culture results $[3,23]$. Many of these issues might be avoided if rapid test methods could be used to reliably detect endoscope channel contamination post-high-level disinfection (HLD) but prior to patient use.

A recent review [24] listed a range of commercially available rapid tests that detect organic residuals (e.g. protein, hemoglobin) and those that detect adenosine triphosphate (ATP) for assessing adequacy of endoscope cleaning. The rapid ATP test kits have been most widely studied for monitoring cleaning adequacy of flexible endoscopes [2,25-27]. Data suggesting cut-offs for adequate cleaning of flexible endoscopes using rapid ATP or organic residuals have been published [25-28]. These cleaning monitoring approaches allow the opportunity to re-clean endoscopes that have not been properly cleaned before they are disinfected. Some authors have questioned whether some of these rapid cleaning monitoring test methods can reliably detect contamination in endoscope channels after HLD [14-15]. There have been very limited assessments of organic residuals post-HLD and clinical studies have reported variable results using the currently available rapid ATP kits to detect potentially contaminated patient-used endoscopes post-disinfection $[10,27,29-31]$. The majority of published studies indicate that the ATP test is too insensitive to detect low levels of viable bacteria in samples extracted from endoscope channels after HLD [2, 10, 25 - 27, 29].

There are few other tests available that reliably detect viable bacteria post-HLD or sterilization prior to patient use. The only other commercially available test for evaluating endoscope channels for viable bacteria is the rapid NOW! (RN) test. The manufacturer's instruction for use (MIFU) indicates that this test can detect as low as 10 CFU of Gram-negative bacteria within endoscope channels. The MIFU indicates the test is not applicable to Gram-positive bacteria as it is based on detection of a specific enzyme found only in Gram-negative bacteria. The sample collected from endoscopes needs to be incubated approximately 12 hours so the MIFU suggests that samples be collected from fully reprocessed endoscopes at the end of the day, incubated overnight and read the following morning before any patient procedures. If there is a test failure detected in the morning, the endoscope can be reprocessed prior to patient use. However (to the best of the authors knowledge), there is only one peer-reviewed publication on this technology [32] and they did not provide specific data for this test in their publication.

Therefore, the objectives of this study were to determine if after overnight incubation the RN test satisfies the 10-CFU limit of detection claim in the MIFU and whether the test can reliably detect low levels of viable Gram-negative bacteria within duodenoscope instrument channels using simulated-use testing.

\section{Materials and methods}

\section{Bacterial strains}

The bacterial strains used included Enterococcus faecalis (ATCC 29212), Escherichia coli (ATCC 25922) and Pseudomonas aeruginosa (ATCC 15442). Each isolate was cultured on blood agar (BA) media and incubated aerobically at $35^{\circ} \mathrm{C}$ for 24 hours prior to use in an experimental test.

\section{Simulated-use study}

Duodenoscopes and automated endoscope reprocessors (AERs)

A TJF-Q180V model (sealed elevator guidewire) Olympus duodenoscope (Olympus Corporation of the Americas, Center Valley, Pennsylvania, United States) was used for simulated-use testing. After each test, the duodenoscope was cleaned as per Olympus MIFU and then processed using the STERIS SYSTEM $1 \mathrm{E}$ (SS1E) (STERIS Inc, Mentor, Ohio, United States) and stored in a channel purge storage cabinet (Torvan Inc, Toronto, Ontario, Canada).

The duodenoscope instrument channel was inoculated by injecting $1 \mathrm{~mL}$ of $10 \%$ blood containing $10^{4} \mathrm{CFU} / \mathrm{mL}$ or $10^{2} \mathrm{CFU} / \mathrm{mL}$ of $E$. coli into the distal opening of the suction channel and then raising the distal end allowing the inoculum to flow towards the biopsy port. The inoculated duodenoscope was allowed to dry for 2 hours prior to sample collection.

\section{Rapid NOW! (RN) test}

\section{Duodenoscope testing}

Samples from the inoculated duodenoscopes were collected by flushing $5 \mathrm{~mL}$ of extraction fluid (sterile water) from the RN test kit through the biopsy port of the instrument channel and the extracted sample was collected from the distal end into a sterile container. A small volume of extraction fluid was used to ensure that the most concentrated channel sample was available for testing. A $0.5-\mathrm{mL}$ portion of the extracted channel sample was inoculated into the test cuvette and incubated overnight as per MIFU. The following day, test reagent was added to the cuvette, which was then placed into the fluorometer and the numeric fluorometer value (NFV) recorded. Baseline testing (i.e. negative endoscope testing) of the fully reprocessed, uninoculated duodenoscope was also done.

The MIFU indicates that an NFV $<200$ indicates no viable Gram-negative bacteria, an NFV 200 to 300 indicates the likely presence of viable Gram-negative bacteria and an NFV > 300 in- 
- Table 1 Simulated-use evaluation of the RN test for detection of E. coli in duodenoscope instrument channel.

\begin{tabular}{|c|c|c|c|c|}
\hline Replicate number & $\begin{array}{l}\text { CFU Inoculum } \\
\text { per channel }\end{array}$ & $\begin{array}{l}\text { Maximum Total CFU } \\
\text { Tested }{ }^{1}\end{array}$ & $\begin{array}{l}\text { NOW! test Incubation } \\
\text { time (Hrs) }\end{array}$ & $\begin{array}{l}\text { Numeric fluorometer } \\
\text { value (NFV) }\end{array}$ \\
\hline \multicolumn{5}{|c|}{ Test 1: Low inoculum } \\
\hline 1 & 320.00 & 32.00 & 21.75 & 2376.00 \\
\hline 2 & 360.00 & 36.00 & 21.67 & 2810.00 \\
\hline 3 & 390.00 & 39.00 & 22.25 & 1832.00 \\
\hline Average: & 356.70 & 35.67 & 21.89 & 2339.33 \\
\hline SD: & 35.12 & 3.51 & 0.31 & 490.03 \\
\hline \multicolumn{5}{|c|}{ Test 2: Moderate inoculum } \\
\hline 1 & 15600.00 & 1560.00 & 20.00 & 2178.00 \\
\hline 2 & 30000.00 & 3000.00 & 20.25 & 4534.00 \\
\hline 3 & 22000.00 & 2200.00 & 21.92 & 4405.00 \\
\hline Average: & 22500.00 & 2253.33 & 21.39 & 3705.67 \\
\hline SD: & 7214.80 & 721.48 & 1.04 & 1324.57 \\
\hline \multicolumn{5}{|c|}{$\begin{array}{l}\text { Bacteria were suspended in ( } 10 \% \text { sheep blood) and the instrument channel of a duodenoscope was inoculated and then allowed to dry for } 2 \text { hours before extractio } \\
\text { as per MIFU (see Materials and Methods for details). The negative control from the test kit showed an NFV of } 0 \text { and } 10 \% \text { blood without any bacteria had an NFV of } 3 \\
{ }^{1} \text { The inoculum per channel was extracted in } 5 \mathrm{~mL} \text { extraction fluid so the maximum CFU tested is calculated as (CFU inoculated } / 5 \mathrm{~mL} \text { ) } \times 0.5 \mathrm{~mL} \text { (volume of extracted } \\
\text { channel sample that is tested). }\end{array}$} \\
\hline
\end{tabular}

dicates presence of viable Gram-negative bacteria. The MIFU indicates that the RN test detects the activity of a specific enzyme found only in viable Gram-negative bacteria and therefore does not detect other types of microorganisms that are not Gram-negative bacteria. The MIFU indicates that the limit of detection for the RN test is 10 CFU in the sample tested.

\section{Suspension testing}

The RN test (Healthmark Industries, Fraser, MI) was evaluated following the MIFU. The limit of detection was assessed using varying separate concentrations of E. faecalis, E. coli and P. aeruginosa prepared in sterile reverse osmosis (sRO) water. A 0.5$\mathrm{mL}$ portion of each concentration of bacteria was inoculated into the test cuvette and incubated overnight as per the MIFU. The following day test reagent was added to the cuvette, which was then placed into the fluorometer and the NFV was recorded.

\section{Viable count for cultures}

The inoculum for suspension testing and also for simulated-use testing were each serially diluted 1:10 using sterile phosphate buffered saline (PBS), and then $0.1 \mathrm{~mL}$ of the direct, as well as each dilution, was spread over the surface of BA plates. Plates were incubated at $35^{\circ} \mathrm{C}$ and colony counts were performed at 24 hours. All tests were performed in triplicate.

\section{Statistical analysis}

The $t$ test was used to assess the area under the Receiver Operator Characteristics (ROC) curve (AUROCC) to determine if AUROCC was significantly different from 0.5 . The greater AUROCC, the more accurate the test. AUROCC of 0.5 suggests that cate- gorization by an evaluated test is similar to random categorization and that the evaluated test is not a useful test. Detection cutoffs of 1 CFU as well as 10 CFU were evaluated. Sensitivity, specificity, positive and negative predictive values (NPV) were calculated.

\section{Results}

The data in $>$ Table 1 are based on simulated-use testing to assess how well the RN test can detect bacteria inoculated into a duodenoscope instrument channel. The RN test MIFU endoscope sample collection consists of a squeezable plastic bottle containing $5 \mathrm{~mL}$ fluid that is flushed through the instrument channel (i.e. does not incorporate friction in the sample extraction protocol). The estimated maximum CFU recoverable in the $0.5-\mathrm{mL}$ samples that were tested by the RN test ranged from 32 to 39 CFU and all three replicates gave a positive test result ( $>$ Table 1 ).

Detection results for the RN test in suspension testing are shown in $>$ Table 2 for E. coli, P. aeruginosa and E. faecalis. As indicated in the MIFU, the Gram-positive bacteria E. faecalis did not generate consistent NFVs over 300 whereas the Gram-negative bacteria tested did have high NFVs even at very low CFU. All nine suspensions with $E$. coli and/or P. aeruginosa $\geq 10 \mathrm{CFU}$ were correctly detected by the RN test. For the cut-off of 1 CFU there was only one of the 24 tests that was incorrect (i. e. P. aeruginosa at 2.35 CFU that was a false-negative). In contrast, for $E$. faecalis, only one of three suspensions with CFU $\geq 10$ tested positive with the RN Test (NFV>300) and none of the three suspensions with CFU between $\geq 1$ and 10 tested positive. 
- Table 2 Performance of the RN test for various bacterial concentrations suspended in sRO water.

Numeric fluorometer value (NFV) for various CFU in suspension

\begin{tabular}{|c|c|c|c|c|c|}
\hline \multicolumn{2}{|l|}{ Test $1^{1}$} & \multicolumn{2}{|l|}{ Test $2^{1}$} & \multicolumn{2}{|l|}{ Test $3^{1}$} \\
\hline Total CFU tested & NFV & Total CFU tested & NFV & Total CFU tested & NFV \\
\hline \multicolumn{6}{|l|}{ Enterococcus faecalis } \\
\hline 13.50 & 178.00 & 20.00 & 331.00 & 15.50 & 43.00 \\
\hline 1.35 & 84.00 & 2.00 & 145.00 & 1.55 & 255.00 \\
\hline 0.14 & 240.00 & 0.20 & 320.00 & 0.16 & 270.00 \\
\hline 0.01 & 193.00 & 0.02 & 121.00 & 0.02 & 246.00 \\
\hline \multicolumn{6}{|l|}{ Escherichia coli } \\
\hline 150.00 & 14373.00 & 225.00 & 15966.00 & 105.00 & 15348.00 \\
\hline 15.00 & 10825.00 & 22.50 & 10052.00 & 10.50 & 11891.00 \\
\hline 1.50 & 8389.00 & 2.25 & 9753.00 & 1.05 & 2109.00 \\
\hline 0.15 & 117.00 & 0.23 & 1.00 & 0.11 & 124.00 \\
\hline \multicolumn{6}{|c|}{ Pseudomonas aeruginosa } \\
\hline 16.00 & 9551.00 & 23.50 & 24196.00 & 22.00 & 10896.00 \\
\hline 1.60 & 13333.00 & 2.35 & 51.00 & 2.20 & 12036.00 \\
\hline 0.16 & 13.00 & 0.24 & 38.00 & 0.22 & 27.00 \\
\hline 0.02 & 70.00 & 0.02 & 0.00 & 0.02 & 5.00 \\
\hline
\end{tabular}

Therefore, sensitivity for detection of CFU $\geq 10$ was $100 \%$ for both $E$. coli and P. aeruginosa and for CFU $\geq 1$ was $100 \%$ for $E$ coli, $83 \%$ for $P$. aeruginosa and $93 \%$ for either. The AUROCC for $E$. coli was 1 for both CFU $\geq 1$ as well as CFU $\geq 10$ and for $P$. aeruginosa was 0.85 for $C F U \geq 10$ and 0.97 for $C F U \geq 1$ ( $P<0.0001)$. Importantly, when the $E$. faecalis suspensions were included as negative controls in the analysis, the sensitivity of NFV $>300$ for detection of CFU $\geq 1$ Gram-negative bacteria was $93 \%$ and specificity was $90 \%$, NPV was $95.0 \%$ and positive predictive value was $88 \%$; the AUROCC was $0.95(P<0.0001)$.

\section{Discussion}

Because it was not feasible to form biofilm within a flexible endoscope, we chose to perform simulated-use testing whereby an organic matrix containing $10 \%$ blood containing low to moderate levels of $E$. coli was used to inoculate a duodenoscope instrument channel that was then allowed to dry for 2 hours. Collecting and testing samples extracted from the inoculated instrument channel confirmed that RN test provided a very strong NFV of over 2300. It should be noted that this signal was generated by a maximum of 32 to 39 CFU in the $0.5 \mathrm{~mL}$ of extracted sample that was used for the RN test. Furthermore, we were able to demonstrate that up to $10 \%$ blood did not interfere with the ability of the test to generate a high NFV.
Our study is the first to demonstrate that in suspension testing, the RN Test does detect very low levels of Gram-negative bacteria but (as stated in the MIFU) cannot reliably detect Gram-positive bacteria. Our data support the manufacturer's claim that the RN test can detect as low as 10 CFU of Gram-negative bacteria in the sample tested. In addition, most of the samples with Gram-negative bacteria CFU between 1 and 10 were also detected as positive by the RN test.

Our previous data showed that for $6639 \mathrm{CFU} / \mathrm{mL}$ of $P$. aeruginosa extracted from biofilm, there was only 1 RLU by the ATP test [9]. Furthermore, it took 7,424,795 CFU/mL extracted from biofilm to generate $1 \mu \mathrm{g} / \mathrm{mL}$ protein [9]. These data demonstrated that although rapid ATP and rapid protein testing are very useful for monitoring manual cleaning compliance, they are very insensitive and cannot act as a substitute for culture post-HLD $[2,10,27,29]$ Unlike rapid ATP and protein testing that do not detect low levels of viable bacteria [9, 23, 25-27, 29] the RN test was sensitive enough to reliably detect nine of nine suspension tests when there were $\geq 10$ CFU of viable $E$. coli and $P$. aeruginosa in the $0.5-\mathrm{mL}$ sample tested. This is an excellent limit of detection but it must be borne in mind that all the current guidelines for interpretation of endoscope cultures [8, 18 - 19] indicate that even 1 CFU per channel of a Gram-negative bacteria is considered unacceptable. Of interest, the RN test correctly detected 14 of 15 of the tests for both of these 
Gram-negative bacteria when there was $\geq 1$ CFU in the $0.5-\mathrm{mL}$ sample tested. All nine suspensions with Gram-negative bacteria $<1$ CFU (i. e. considered as a negative sample) had NFV readings $<300$. Although there are a number of Gram-positive bacteria that are organisms of concern [8,18-19], there have been no infectious outbreaks attributed to such bacteria arising from contaminated endoscopes, so utilizing a test that only detects Gram-negative organisms of concern maybe a reasonable alternative option to routine universal culturing. Potential advantages of RN test compared to cultures are a more rapid test (overnight compared with 48- to 72-hour hold for cultures), lack of need for addition of a neutralizer and concentration of the samples (neutralizer and sample concentration increase sensitivity of cultures but have no role with RN test) and ability to process in the endoscopy unit itself (compared with cultures which require transportation to a Microbiology laboratory).

Although $10 \%$ blood did not generate a NFV ( $\vee$ Table 1 ), it may affect the optical NFV reading as the signal generated from samples with $10 \%$ blood ( $\triangleright$ Table $1 ; 2,253.33$ CFU of E. coli had NFV of 3,705) were not as high as those generated when the same organism was in sRO water ( $>$ Table 2; 105 CFU E. coli had NFV of 15,348). This aspect did not affect the ability of the RN test to detect low levels of viable Gram-negative bacteria, however, further assessment of the effect of organic residuals on the RN test is needed.

A limitation of this study is that we only evaluated two Gram-negative bacteria so further studies to show the applicability of the RN test for a wider range of Gram-negative bacteria would be valuable. The MIFU does not indicate the extraction efficacy of the 5-mL flush with extraction fluid. In addition, we did not evaluate the extraction efficacy of flushing $5 \mathrm{~mL}$ through an instrument channel containing dried organic debris, biofilm or build-up biofilm. Future clinical studies are needed to evaluate the feasibility of implementation of the RN test for patient-used endoscopes in a busy endoscopy clinic. The RN test cannot be used for testing endoscopes used more than once during the day as this test requires overnight incubation. In addition, it is unclear if testing endoscopes used on a Friday would require reading of $\mathrm{RN}$ test results on Saturday or whether the test could still provide reliable results if the completion of testing was done Monday morning. Despite these limitations, the $\mathrm{RN}$ test is the only currently available test that can provide reliable detection of 1 to 10 CFU with an overnight turnaround time. Our data support the recent study by Washburn et al. who reported that the $\mathrm{RN}$ test had similar results compared to culture [32].

\section{Conclusion}

In conclusion we have demonstrated that the RN test can reliably detect $\geq 10$ CFU (and most of $\geq 1$ CFU) of Gram-negative bacteria which is superior to what current rapid ATP and protein test kits can detect. Our encouraging preliminary data suggest that the RN test may be a useful alternative to culture for assessing contamination with Gram-negative bacteria in patientready endoscopes but further evaluation of extraction efficiency, effect of organic residuals and clinical testing is needed.

\section{Acknowledgements}

Funding for this study was provided by a research grant from the American Society for Gastrointestinal Endoscopy (ASGE). The Olympus duodenoscope, as well as the EFP-500 flushing pump were loaned and the channel and cavity brushes were donated by Olympus America. The endoscope storage cabinet was donated by Torvan Inc. The RN test kits were donated by Healthmark Industries.

\section{Competing interests}

Harminder Singh: Advisory Board;Ferring Canada \& Takida Canada, Research funds; Merck Canada

Michelle Alfa: Advisory board;3M, J\&], Consultant for Olympus, Novaflux, Ofstead Associates, Royalty through University of Manitoba for license to HealthMark Industry for ATS test soil patent, Invited speaker for Olympus, 3M, Ambu \& Sealed Air

Donald Duerksen: Advisory Board for Ferring Canada

All other authors have no conflicts of interest

References

[1] Epstein L, Hunter JC, Arwady MA et al. New Delhi metallo-beta-lactamase-producing carbapenem-resistant Escherichia coli associated with exposure to duodenoscopes. JAMA 2014; 312: 1447 - 1455

[2] Visrodia K, Hanada Y, Pennington KM et al. Duodenoscope reprocessing surveillance with adenosine triphosphate testing and terminal cultures: a clinical pilot study. Gastrointest Endosc 2017; 86: 180 186

[3] Ross AS, Baliga C, Verma P et al. A quarantine process for the resolution of duodenoscope-associated transmission of multidrug-resistant Escherichia coli. Gastrointest Endosc 2015; 82: 477 - 483

[4] Wendorf KA, Kay M, Baliga C et al. Endoscopic retrograde cholangiopancreatography-associated AmpC Escherichia coli outbreak. Infect Control Hosp Epidemiol 2015; 36: 634-642

[5] Kovaleva J, Peters FT, van der Mei HC et al. Transmission of infection by flexible gastrointestinal endoscopy and bronchoscopy. Clin Microbiol Rev 2013; 26: $231-254$

[6] Ma GK, Pegues DA, Kochman ML et al. Implementation of a systematic culturing program to monitor the efficacy of endoscope reprocessing: outcomes and costs. Gastrointest Endosc 2018; 87: 104 - 109 e3

[7] US Food and Drug Administration. Supplemental measures to enhance duodenoscope reprocessing: FDA safety communication. Accessed November 23, 2018. Available at: https://www.fdanews.com/ ext/resources/files/08-15/081015-duodenoscopes-fda.pdf? 1520541508

[8] US Food \& Drug Administration, Centers for Disease Control and Prevention, American Society for Microbiology. Duodenoscope surveillance sampling \& culture: Reducing the risks of infection. Accessed November 23, 2018. Available at: https://www.fda.gov/downloads/ MedicalDevices/ProductsandMedicalProcedures/ReprocessingofReusableMedicalDevices/UCM597949.pdf

[9] Alfa M], Singh H, Nugent Z et al. Sterile reverse osmosis water combined with friction are optimal for channel and lever cavity sample collection of flexible duodenoscopes. Front Med (Lausanne) 2017; 4: 191 
[10] Cattoir L, Vanzieleghem T, Florin L et al. Surveillance of endoscopes: comparison of different sampling techniques. Infect Control Hosp Epidemiol 2017; 38: 1062 - 1069

[11] Saliou P, Le Bars H, Payan C et al. Measures to improve microbial quality surveillance of gastrointestinal endoscopes. Endoscopy 2016; 48: $704-710$

[12] Saviuc P, Picot-Gueraud R, Shum Cheong Sing J et al. Evaluation of the quality of reprocessing of gastrointestinal endoscopes. Infect Control Hosp Epidemiol 2015; 36: 1017-1023

[13] Gazdik MA, Coombs J, Burke JP et al. Comparison of two culture methods for use in assessing microbial contamination of duodenoscopes. J Clin Microbiol 2016; 54: 312 - 316

[14] Ofstead CL, Wetzler HP, Eiland JE et al. Assessing residual contamination and damage inside flexible endoscopes over time. Am J Infect Control 2016; 44: 1675-1677

[15] Ofstead CL, Wetzler HP, Heymann OL et al. Longitudinal assessment of reprocessing effectiveness for colonoscopes and gastroscopes: Results of visual inspections, biochemical markers, and microbial cultures. Am J Infect Control 2017; 45: e26 - e33

[16] Shin SP, Kim WH. Recent update on microbiological monitoring of gastrointestinal endoscopes after high-level disinfection. Clin Endosc 2015; 48: 369-373

[17] Paula H, Presterl E, Tribl B et al. Microbiologic surveillance of duodenoscope reprocessing at the Vienna University Hospital from November 2004 through March 2015. Infect Control Hosp Epidemiol 2015; 36: $1233-1235$

[18] Beilenhoff U, Neumann CS, Rey JF et al. ESGE-ESGENA guideline for quality assurance in reprocessing: microbiological surveillance testing in endoscopy. Endoscopy 2007; 39: 175- 181

[19] Taylor A, Jones D, Everts R et al. Infection control in endoscopy. Third Edition ed. Victoria, Australia: Gastroenterological Society of Australia; 2010

[20] Instrumentation AftAoM. Flexible and semi-rigid endoscope processing in health care facilities (ANSI/AAMI ST91: 2015). Arlington, VA: Author 2015.

[21] Petersen BT, Cohen J, Hambrick RD et al. Multisociety guideline on reprocessing flexible $\mathrm{Gl}$ endoscopes: 2016 update. Gastrointestinal Endoscopy 2017; 85: 282-294.e1
[22] Herrin A, Loyola M, Bocian S et al. Standards of infection prevention in reprocessing flexible gastrointestinal endoscopes. Gastroenterol Nurs 2016; 39: $404-418$

[23] Alfa MJ, Ribeiro MM, da Costa Luciano C et al. A novel polytetrafluoroethylene-channel model, which simulates low levels of culturable bacteria in buildup biofilm after repeated endoscope reprocessing. Gastrointest Endosc 2017; 86: 442 - 451 e1

[24] Komanduri S, Abu Dayyeh BK, Bhat YM et al. Technologies for monitoring the quality of endoscope reprocessing. Gastrointest Endosc 2014; 80: 369-373

[25] Alfa MJ, Fatima I, Olson N. The adenosine triphosphate test is a rapid and reliable audit tool to assess manual cleaning adequacy of flexible endoscope channels. Am J Infect Control 2013; 41: 249-253

[26] Alfa MJ, Fatima I, Olson N. Validation of adenosine triphosphate to audit manual cleaning of flexible endoscope channels. Am J Infect Control 2013; 41: 245-248

[27] Parohl N, Stiefenhofer D, Heiligtag S et al. Monitoring of endoscope reprocessing with an adenosine triphosphate (ATP) bioluminescence method. GMS Hyg Infect Control 2017; 12: Doc04

[28] Alfa M], Olson N, Degagne P et al. Development and validation of rapid use scope test strips to determine the efficacy of manual cleaning for flexible endoscope channels. Am J Infect Control 2012; 40: 860 865

[29] Olafsdottir LB, Wright SB, Smithey A et al. Adenosine triphosphate quantification correlates poorly with microbial contamination of duodenoscopes. Infect Control Hosp Epidemiol 2017; 38: 678-684

[30] Gillespie EE, Kotsanas D, Stuart RL. Microbiological monitoring of endoscopes: 5-year review. J Gastroenterol Hepatol 2008; 23: 10691074

[31] Sethi S, Huang RJ, Barakat MT et al. Adenosine triphosphate bioluminescence for bacteriologic surveillance and reprocessing strategies for minimizing risk of infection transmission by duodenoscopes. Gastrointest Endosc 2017; 85: 1180-1187 e1

[32] Washburn RE, Pietsch JJ. Assessment of test methods for evaluating effectiveness of cleaning flexible endoscopes. Am J Infect Control 2018; 46: 685-688 